Research Article

\title{
Signal Modulation Identification Based on Deep Learning in FBMC/OQAM Systems
}

\author{
Jing Chen, ${ }^{1}$ Jianzhong Guo, ${ }^{2}$ Xin Shan, ${ }^{1}$ and Dejin Kong $\mathbb{D D}^{2}$ \\ ${ }^{1}$ Electronic Information School, Wuhan University, Wuhan 430072, China \\ ${ }^{2}$ School of Electronic and Electrical Engineering, Wuhan Textile University, Wuhan 430200, China \\ Correspondence should be addressed to Dejin Kong; djkou@wtu.edu.cn
}

Received 6 August 2021; Accepted 27 September 2021; Published 14 October 2021

Academic Editor: Han Wang

Copyright (c) 2021 Jing Chen et al. This is an open access article distributed under the Creative Commons Attribution License, which permits unrestricted use, distribution, and reproduction in any medium, provided the original work is properly cited.

Signal modulation identification (SMI) has always been one of hot issues in filter-bank multicarrier with offset quadrature amplitude modulation (FBMC/OQAM), which is usually implemented by the machine learning-based feature extraction. However, it is difficult for conventional methods to extract the signal feature, resulting in a limited probability of correct classification (PCC). To tackle this problem, we put forward a novel SMI method based on deep learning to identify FBMC/ OQAM signals in this paper. It is noted that the block repetition is employed in the FBMC/OQAM system to achieve the imaginary interference cancelation. In the proposed deep learning-based SMI technique, the in-phase and quadrature samples of FBMC/OQAM signals are trained by the convolutional neural network. Subsequently, the dropout layer is designed to prevent overfilling and improve the identification accuracy. To evaluate the proposed scheme, extensive experiments are conducted by employing datasets with different modulations. The results show that the proposed method can achieve better accuracy than conventional methods.

\section{Introduction}

Filter-bank multicarrier with offset quadrature amplitude modulation (FBMC/OQAM) has been considered as one of the potential physical-layer techniques for future wireless communications [1-4]. Owing to the use of pulse shaping filter with low spectrum sidelobe, FBMC/OQAM offers the high spectrum utilization and good ability of asynchronous transmission $[1,2]$. In addition, the cyclic prefix is not required in FBMC/OQAM, leading to a high spectral efficiency. However, in contrast to the classical orthogonal frequency division multiplexing (OFDM), the orthogonality of FBMC/OQAM only holds in the real-valued field. To meet the orthogonality condition, FBMC/OQAM systems transmit real-valued symbols obtained by the real and imaginary parts of complex-valued QAM symbols, and there exist imaginary interferences among the transmitted realvalued symbols, called the intrinsic imaginary interference [3]. The imaginary interference will make a major effect on the algorithms about the parameter estimation, which has to be considered in the algorithm design.

With the rapid development of wireless communications, noncooperative communications will be very common in military and civilian areas. In order to identify different modulations of the received signals, signal modulation identification (SMI) is required in the noncooperation wireless communications $[5,6]$. For instance, diverse eavesdropping risks can be identified in wireless links by the SMI technique, which ensure the system integrity [7]. In the electronic countermeasure, the signal modulation type is first required to estimate from the intercepted electromagnetic waves. On this basis, the intercepted signal could be decrypted further [8]. Therefore, SMI has been regarded as one of the most crucial techniques when we design a noncooperative communication system. Developing the accurate SMI method is necessary to identify FBMC/OQAM signals [9] since the SMI design is a big challenge to identify types of FBMC/OQAM signals. 
So far, most existing modulation identification approaches are based on feature extraction and machine learning classification. A lot of SMI schemes are developed by combining different machine learning-based classifiers and feature extraction strategies [10]. However, since conventional feature extraction schemes rely on statistics, it is difficult for these methods to extract the signal features of different modulation types. As a result, the classification result is vulnerable to mix up. In addition, machine learningbased SMI schemes will suffer performance bottleneck for the problem associated with big data. That is to say, the probability of correct classification is not good enough, which indicates unpreferred in practical FBMC/OQAM systems. To solve this issue, deep learning (DL) has been deemed to be one of effective techniques to deploy SMI [11]. In [12], an enhanced identification scheme was presented based on deep neural network (DNN). In [13], the genetic programming (GP) was combined with the $k$-nearest neighbor (KNN) to identify four modulation types accurately.

In this paper, we propose a novel SMI technique based on the convolutional neural network $(\mathrm{CNN})$ to make an identification on FBMC/OQAM signals. It is noted that the block repetition is employed in the FBMC/OQAM system to achieve the imaginary interference cancelation. In the proposed scheme, 3 fully connected layers and 2 convolutional layers are designed. In addition, the dropout layer is designed to lower the interaction neurons of the same layer. To evaluate the proposed scheme, extensive experiments are conducted by employing datasets with different modulations. The results show that the proposed method can achieve better accuracy than conventional methods.

The rest of the paper is organized as follows. The system model of FBMC/OQAM with block repetition is introduced briefly, as well as the model of the deep learning in Section 2. Subsequently, the proposed SMI method is presented in Section 3. Section 4 gives experimental results, followed by the conclusion in Section 5.

\section{Model of FBMC/OQAM}

Figure 1 depicts the baseband FBMC/OQAM system diagram with $M$ subcarriers. The transmitted signal of each FBMC/OQAM subcarrier gets through a pulse shaping filter. $d_{m, n}$ is the transmitted symbol at the time frequency position $(m, n)$. Denote $g[l]$ as the pulse shaping filter for all subcarriers in FBMC/OQAM, which has the even and symmetric coefficients and exhibits the extremely low spectrum sidelobe [2]. According to Figure 1, the transmitted signal of FBMC/OQAM can be expressed as follows $[3,14]$ :

$$
s[l]=\sum_{m=0}^{M-1} \sum_{n \in \mathbb{Z}} d_{m, n} g\left[l-n \frac{M}{2}\right] e^{((j 2 \pi m l) / M)} e^{((j \pi(m+n)) / 2)} .
$$

Assume $h[l]$ is the Rician fading channel. The transmitted signal $s[l]$ passes through the channel $h[l]$, and the received signal can be written as

$$
r[l]=h[l] * s[l]+\eta[l],
$$

where * represents the convolution operator and $\eta[l]$ stands for the additive white Gaussian noise [15].

Then, after FBMC/OQAM demodulation at the receiver, it can obtain the following $[16,17]$ :

$$
\begin{aligned}
\widehat{d}_{m, n} & =\sum_{l=-\infty}^{\infty} r[l] g\left[l-n \frac{M}{2}\right] e^{-(j 2 \pi m l / M)} e^{-(j \pi(m+n) / 2)} \\
& =H_{m, n}\left(d_{m, n}+\sum_{\left(m_{0}, n_{0}\right) \neq(m, n)} d_{m_{0}, n_{0}} \xi_{m, n}^{m_{0}, n_{0}}\right)+\eta_{m, n} \\
& =H_{m, n}\left(d_{m, n}+j d_{m, n}^{c}\right)+\eta_{m, n},
\end{aligned}
$$

where $\zeta_{m, n}^{m_{0}, n_{0}}$ is the imaginary interference factor in FBMC/ OQAM, which is a pure imaginary value, $d_{m, n}^{c}$ is the imaginary interference to the symbol $d_{m, n}$ [17], and $\eta_{m, n}$ is the noise to the symbol $d_{m, n}$. Note that, although $\eta[l]$ is white Gaussian noise, $\eta_{m, n}$ is not white due to the real-valued orthogonality of FBMC/OQAM systems. $H_{m, n}$ is the channel frequency response at the subcarrier $m$. For the time-invariant channels, the value of $H_{m, n}$ is not relevant to the time index $n$.

It has been demonstrated that the imaginary interference factor $\zeta_{m, n}^{m_{0}, n_{0}}$ is symmetrical [18]. On this basis of symmetric, the block repetition is designed in FBMC/OQAM to achieve the imaginary interference cancelation $[18,19]$. As shown in Figure 2, the frame consists of two parts, i.e., original block and repeated block. $d_{m, n} m \in[0, M-1], n \in[0, N-1]$ are the symbols in the original block, which are obtained from the QAM constellation. $a_{m, n+N}$ are the symbols in the repeated block, and it is noted that $a_{m, n+N}=a_{m, N-1-n}$.

At the receiver, the demodulations of original block and repeated block are, respectively, written as

$$
\widehat{d}_{m, n}=H_{m, n}\left(d_{m, n}+j d_{m, n}^{c}\right)+\eta_{m, n}, \quad n=0,1, \ldots, N-1,
$$

$$
\begin{aligned}
\widehat{d}_{m, 2 N-1-n}= & H_{m, 2 N-1-n}\left(d_{m, 2 N-1-n}+j d_{m, 2 N-1-n}^{c}\right) \\
& +\eta_{m, 2 N-1-n} \\
= & H_{m, n}\left(d_{m, n}+j d_{m, 2 N-1-n}^{c}\right) \\
& +\eta_{m, 2 N-1-n}, \quad n=0,1, \ldots, N-1 .
\end{aligned}
$$

Note that, the imaginary interferences of the original block and repeated block satisfy the following equation [18], i.e.,

$$
j d_{m, n}^{c}+j d_{m, 2 N-1-n}^{c}=0 .
$$

Subsequently, the imaginary interferences can be removed by the following linear combination:

$$
\frac{\widehat{d}_{m, n}+\widehat{d}_{m, 2 N-1-n}}{2}=H_{m, n} d_{m, n}+\frac{\eta_{m, n}+\eta_{m, 2 N-1-n}}{2} .
$$




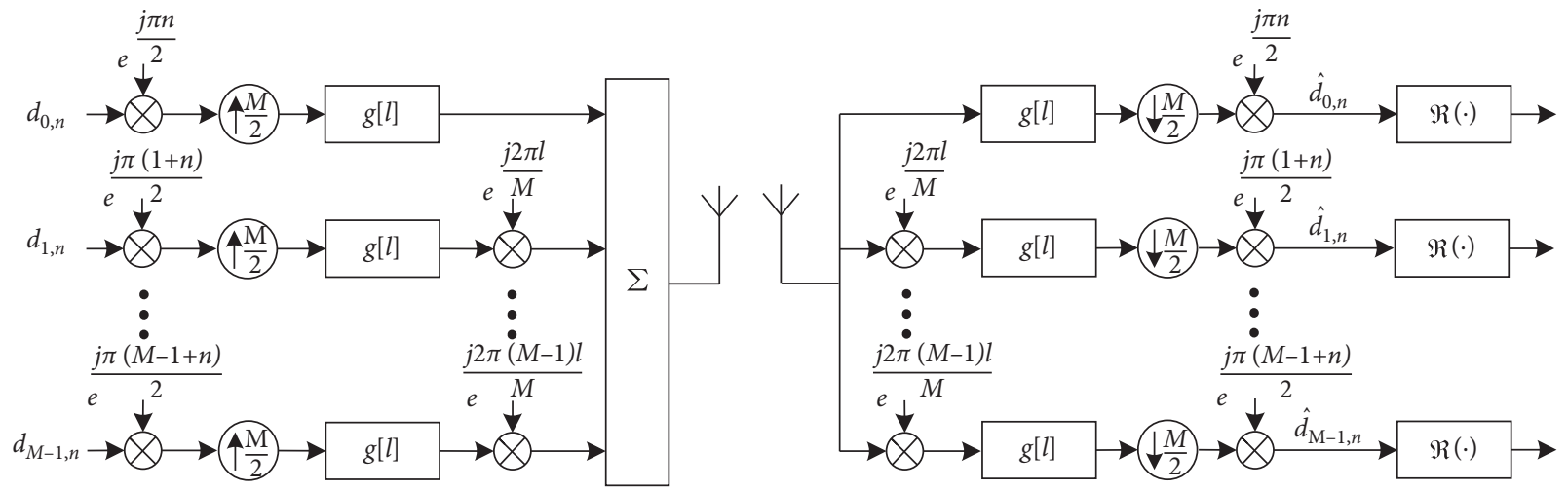

Figure 1: The FBMC/OQAM system diagram.

\begin{tabular}{|c|c|c|c|c|c|c|c|c|}
\hline$d_{0,0}$ & $d_{0,1}$ & $d_{0,2}$ & $d_{0,3}$ & $d_{0,4}$ & $d_{0,4}$ & $d_{0,3}$ & $d_{0,2}$ & $d_{0,1}$ \\
\hline$d_{1,0}$ & $d_{1,1}$ & $d_{1,2}$ & $d_{1,3}$ & $d_{1,4}$ & $d_{1,4}$ & $d_{1,3}$ & $d_{1,2}$ & $d_{1,1}$ \\
\hline$d_{2,0}$ & $d_{2,1}$ & $d_{2,2}$ & $d_{2,3}$ & $d_{2,4}$ & $d_{2,4}$ & $d_{2,3}$ & $d_{2,2}$ & $d_{2,1}$ \\
\hline$d_{3,0}$ & $d_{3,1}$ & $d_{3,2}$ & $d_{3,3}$ & $d_{3,4}$ & $d_{3,4}$ & $d_{3,3}$ & $d_{3,2}$ & $d_{3,1}$ \\
\hline$d_{4,0}$ & $d_{4,1}$ & $d_{4,2}$ & $d_{4,3}$ & $d_{4,4}$ & $d_{4,4}$ & $d_{4,3}$ & $d_{4,2}$ & $d_{4,1}$ \\
\hline$d_{5,0}$ & $d_{5,1}$ & $d_{5,2}$ & $d_{5,3}$ & $d_{5,4}$ & $d_{5,4}$ & $d_{5,3}$ & $d_{5,2}$ & $d_{5,1}$ \\
\hline
\end{tabular}

Figure 2: The reversed-order block for FBMC/OQAM.

Let $\bar{d}_{m, m}=\left(\widehat{d}_{m, n}+\widehat{d}_{m, 2 N-1-n}\right) / 2$ and $\bar{\eta}_{m, n}=\left(\eta_{m, n}+\right.$ $\left.\eta_{m, 2 N-1-n}\right) / 2$. Then, equation (7) can be rewritten as

$$
\bar{d}_{m, m}=H_{m, n} d_{m, n}+\bar{\eta}_{m, n} .
$$

Note that, the noise $\bar{\eta}_{m, n}$ satisfies the white Gaussian distribution [18].

Suppose the Rician fading channel only consists of a line of sight (LOS) between the receiver and the transmitter. Then, the probability of correct classification (PCC) of the Rician distribution is written as

$$
f[x]=\frac{x}{\sigma^{2}} e^{-\left(x^{2}+A^{2} / 2 \sigma^{2}\right)} \cdot I_{0}\left[\frac{A x}{\sigma^{2}}\right],
$$

where $\sigma^{2}$ stands for the power of the multipath signal component, $A$ represents the amplitude peak of the main signal, $I_{0}[\cdot]$ stands for the modified Bessel function of the 0 th order. The Rician channel model can be written as

$$
h[l]=\sqrt{\frac{\kappa}{\kappa+1}} \sigma e^{j \theta}+\sqrt{\frac{1}{\kappa+1}} \mathbb{N}\left(0, \sigma^{2}\right),
$$

where the first part stands for the mirror path which has the uniform phase $\theta$. The second part represents a majority of scattering paths and reflection paths, which are independent of $\theta . \kappa$ is the Rician factor that indicates the Rician distribution. The Rician factor is defined as

$$
\kappa=\frac{A x}{\sigma^{2}} .
$$

When the factor $\kappa$ goes to zero gradually, the Rician distribution will become a Rayleigh distribution.

\section{Proposed Deep Learning-Based SMI}

3.1. CNN Algorithm. After converting to IQ samples, the FBMC/OQAM signal can be used to train the CNN. As a commonly used algorithm, $\mathrm{CNN}$ is one of hot research issues in the area of artificial intelligence. The $\mathrm{CNN}$ structure mainly includes input layers, convolution layer, pooling layer, fully connected layer, and output layer. The main features of $\mathrm{CNN}$ are summarized as follows:

(i) Local Connection. For this connection, each neuron is not required to connect the whole neurons from the upper layer but only a small part of neurons, which can reduce the parameters significantly.

(ii) Weight Sharing. It not required that each connection corresponds to one weight. Instead, one set of connections has the same weight, which is also beneficial to reduce the number of parameters.

(iii) Down Sampling. The number of samples for each layer can be reduced by the pooling layer, which can enhance the model robustness.

In the CNN structure, the convolution layer is the most important part, which can be compared with the convolution operation in calculus. For instance, the convolutional signal between a time-domain signal $x[k]$ and a time-domain signal $w[k]$ can be written as

$$
y[k]=\sum_{l} x[k-l] w[l] .
$$

For the two-dimension signals $x[p, q]$ and $w[p, q]$, the convolutional signal can be written as

$$
y[p, q]=\sum_{k} \sum_{l} x[p-k, q-l] w[k, l]
$$

It is noteworthy that the convolution formula in the $\mathrm{CNN}$ algorithm has a difference slightly with the abovementioned convolution definition. For example, the twodimensional convolution in $\mathrm{CNN}$ is written as 


$$
y[p, q]=\sum_{k} \sum_{l} x[p+k, q+l] w[k, l] .
$$

3.2. Deep Learning-Based SMI Method. In this subsection, the deep learning-based SMI method is proposed via the CNN algorithm, in which 2 convolutional layers and 3 fully connected layers are included, as shown in Figure 3. Specifically, the first one of convolutional layers consists of 128 convolution kernels with the $1 \times 16$ dimension matrix for every convolution kernel. As for the second one of convolutional layers, there exist 64 convolution kernels with the $2 \times 8$ dimension matrix for every convolution kernel. The neuron numbers of the 3 fully connected layers are 256, 128, and $\lambda$, respectively. Note that, $\lambda$ denotes the number of the modulation modes used in FBMC/OQAM systems. In addition, except the last one of 3 fully connected layers, the activation function of all layers is the parametric rectified linear unit (PReLU), which can effectively reduce the problem of the gradient disappearance during the operation of back-propagation. As for the last one of 3 fully connected layers, softmax is taken to acquire the probability distribution matrix.

In addition, the dropout layer is added in the first 4 layers in the proposed deep learning-based SMI structure to decrease the overfitting effectively. In a circle, some neurons are randomly selected in the neural layer and are hidden temporarily. Subsequently, the training process of the CNN is carried out. In the next circle, some other neurons will be hidden until the training ends. Note that, the proposed scheme can decrease the interaction among neurons, which makes the technique more generalizable.

3.3. Dataset. To verify the proposed method, two datasets are created for the task of SMI. The modulations in dataset $\theta_{1}$ consist of BPSK, QPSK, 8PSK, and 16QAM, and the modulations in dataset $\theta_{2}$ include BPSK, QPSK, 8PSK, 16QAM, and 64QAM. For the training and testing of CNN, 40000 data samples are created for each modulation. For example, for a certain signal-to-noise ratio (SNR), there are 160000 data samples that are input into the neural network. Note that, the samples for training take up $70 \%$ of the whole samples and the samples for testing take up 30\% of the whole samples.

(a) IQ Samples. Through the FBMC/OQAM modulation and the channel, the $i$-th samples can be obtained as

$$
S_{i}=\left[s_{0}, s_{1}, \ldots, s_{N_{s}}\right] \text {, }
$$

where $N_{s}$ stands for the number of samples and $s_{l}$ is the value of the $l$-th sample. Since $s_{l}$ is a complexvalued number, it can be rewritten as

$$
s_{l}=\mathfrak{R}\left\{s_{l}\right\}+j \mathfrak{\Im}\left\{s_{l}\right\},
$$

where $\mathfrak{R}\{\cdot\}$ and $\mathfrak{I}\{\cdot\}$ represent the operations of taking real and imaginary parts, respectively. $\mathfrak{R}\left\{s_{l}\right\}$ and $\mathfrak{\Im}\left\{s_{l}\right\}$ stand for the in-phase component and quadrature component of the signal.

(b) AP Samples. The module and phase of $s_{l}$ can be obtained as

$$
\begin{aligned}
& A=\sqrt{\mathfrak{R}\left\{s_{l}\right\}^{2}+\Im_{\{}\left\{s_{l}\right\}^{2}}, \\
& \theta=\arctan \frac{\mathfrak{\Im}\left\{s_{l}\right\}}{\mathfrak{R}\left\{s_{l}\right\}}
\end{aligned}
$$

(c) Manmade Features. The higher order cumulants (HOC) feature can be calculated, and the manmade feature can be constituted by combining the instantaneous feature of the signal.

\section{Experiment Results}

In this section, experiments are conducted to verify the performance of the proposed deep learning-based SMI technique in the noncooperative FBMC/OQAM system. We also give compare the identification accuracy with conventional methods. In the experiments, 256 subcarriers are considered in the FBMC/OQAM system, and only 16 subcarriers are active. In addition, each subcarrier contains 8 symbols. As for the Rician channel, the Rician factor is 20 and the sampling frequency is $10 \mathrm{kHz}$, suffering from a Doppler frequency offset $400 \mathrm{~Hz}$. In the experiment, 3 existing classification schemes are used for the comparison, which are all machine learning-based methods, i.e., CNN with IQ samples, CNN with AP samples, and deep neural network (DNN) with manmade features extracted by logistic regressive.

Figure 4 depicts the PCC comparison between the proposed scheme and the existing schemes, in which dataset $\theta_{1}$ is used for training. From the results, the PCC of the proposed scheme is improving gradually, while the existing methods remain unchanged almost. When $\mathrm{SNR}>20 \mathrm{~dB}$, the accuracy of modulation identification nearly achieves $100 \%$ by the proposed deep learning-based SMI technique. In addition, the CNN with AP samples can achieve better PCC accuracy than the DNN method with manmade features which is because that CNN can extract the data features automatically via the convolution kernel.

As shown in Figure 5, the PCCs for different modulation modes are depicted by the CNN with IQ samples. From the results, the BPSK modulation can be always identified correctly at the whole range of the SNR, while the other three modulations suffer the accuracy degradation at the SNR range below $15 \mathrm{~dB}$. When the SNR comes to the high SNR range, the PCCs of all modulations stabilize gradually. Above all, the proposed technique can achieve high PCC accuracy for the signal modulation identification.

In Figures 4 and 5, dataset $\theta_{1}$ is used for the signal identification to evaluate the proposed SMI technique. However, the performance may be affected by the introduction of a new modulation in practice. To demonstrate the robustness of the proposed scheme, dataset $\theta_{2}$ is used for the causing the dataset mismatch. Figure 6 shows the PCCs of 


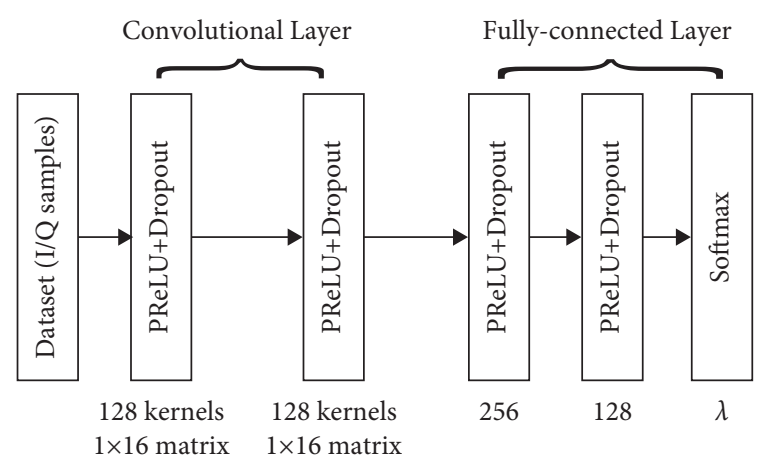

FIgURE 3: The structure of deep learning-based SMI.

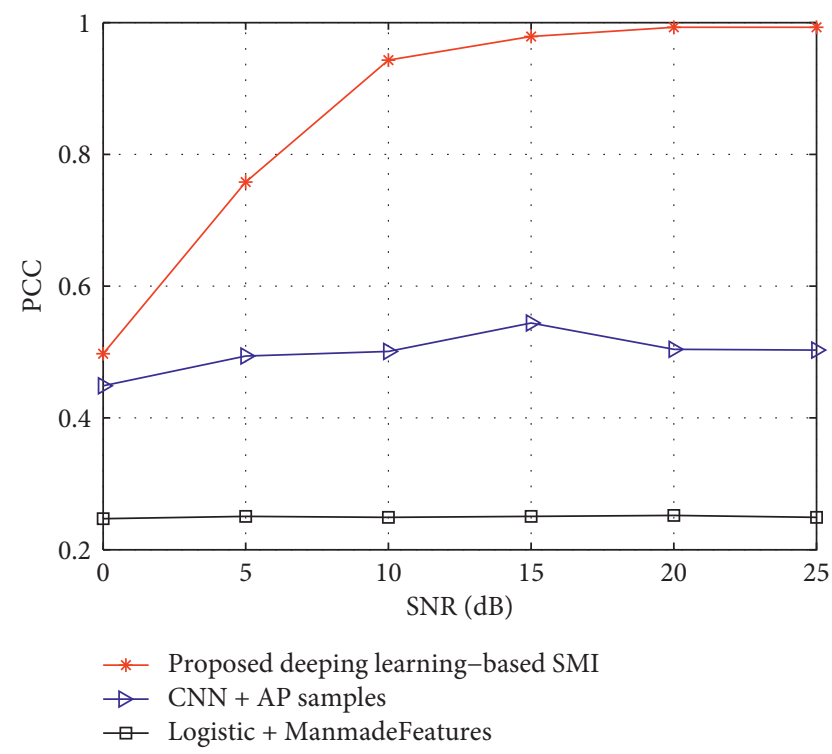

FIGURE 4: PCC comparison between the proposed scheme and the existing schemes, dataset $\theta_{1}$.

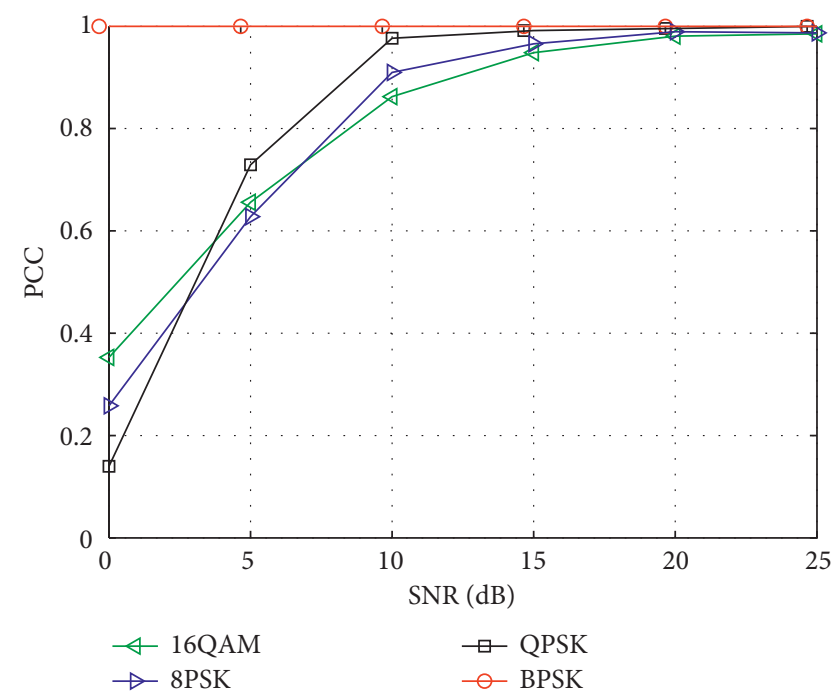

FIGURE 5: PCC comparison between different modulation types, dataset $\theta_{1}$.

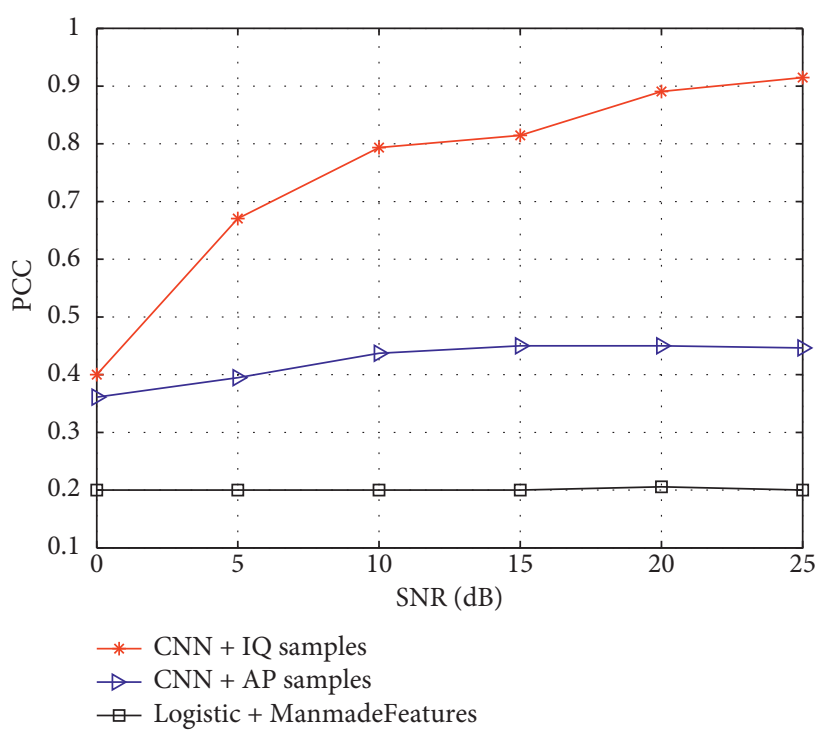

Figure 6: PCC comparison of different methods, dataset $\theta_{2}$.

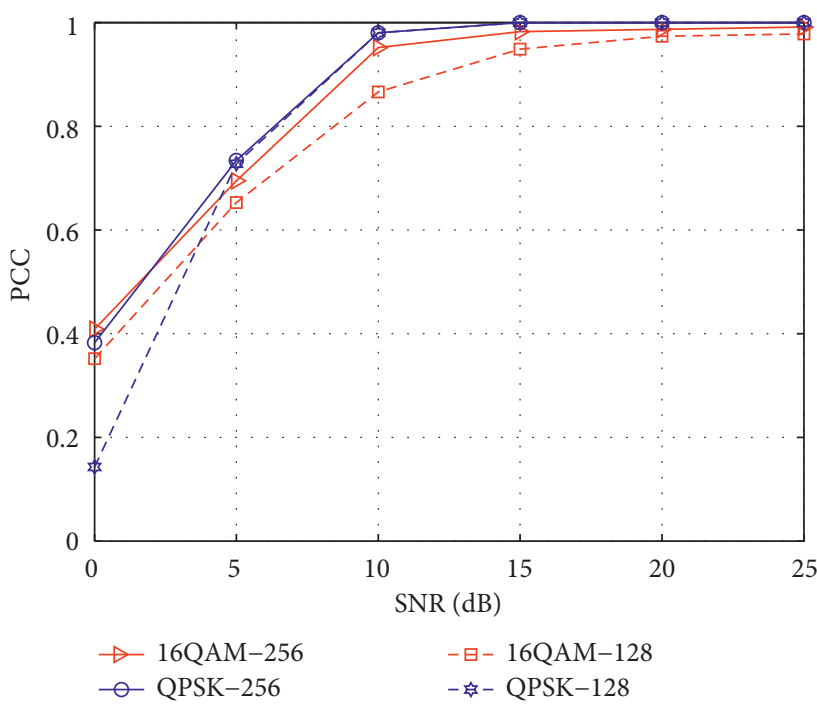

FIGURE 7: PCC comparison of the proposed scheme under different sample points, dataset $\theta_{1}$.

the proposed scheme under the dataset mismatch. From the result, the curve trend of Figure 6 is similar to that of Figure 4, and the PCC accuracy remains steady in the proposed scheme, achieving the accuracy of above $90 \%$. The mismatch of the datasets has no damage on the signal modulation identification.

In the following experiments, the sample points of the signal are increased to verify the proposed deep learningbased SMI technique. Figure 7 depicts the PCC comparison of the proposed scheme under different sample points, in which dataset $\theta_{1}$ is employed. From the results, the proposed scheme with 256 points can achieve higher PCC accuracy than the method with 128 sample points. Especially, the performance difference is more obvious at low SNR. The reason is that, as the sample points increase, more features of 
the signal can be extracted by the CNN algorithm, improving the identification accuracy effectively.

\section{Conclusions}

In this paper, a deep learning-based SMI technique was presented to identify FBMC/OQAM signals in a noncooperative system. To achieve the imaginary interference cancelation, the concept of block repetition was employed in the FBMC/OQAM system to generate signals. In the proposed scheme, 3 fully connected layers and 2 convolutional layers were designed. In addition, the dropout layer was designed to lower the interaction neurons of the same layer. To evaluate the proposed scheme, extensive experiments were conducted by employing datasets with different modulations. The results indicated that the proposed deep learning-based SMI scheme exhibited high accuracy of modulation identification and strong robustness.

\section{Data Availability}

The data used to support the findings of this study are available from the corresponding author upon request.

\section{Conflicts of Interest}

The authors declare that there are no conflicts of interest regarding the publication of this paper.

\section{Acknowledgments}

This work was financially supported in part by the Scientific Research Fund of Wuhan Textile University under Grant 20200828.

\section{References}

[1] R. Nissel, S. Schwarz, and M. Rupp, "Filter bank multicarrier modulation schemes for future mobile communications," IEEE Journal on Selected Areas in Communications, vol. 35, no. 8, pp. 1768-1782, 2017.

[2] P. Siohan, C. Siclet, and N. Lacaille, "Analysis and design of OFDM/OQAM systems based on filterbank theory," IEEE Transactions on Signal Processing, vol. 50, no. 5, pp. 11701183, 2002.

[3] D. Kong, D. Qu, and T. Jiang, "Time domain channel estimation for OQAM-OFDM systems: algorithms and performance bounds," IEEE Transactions on Signal Processing, vol. 62, no. 2, pp. 322-330, 2014.

[4] R. Nissel and M. Rupp, "Pruned DFT-spread FBMC: low PAPR, low latency, high spectral efficiency," IEEE Transactions on Communications, vol. 66, no. 10, pp. 4811-4825, 2018.

[5] F. Wen, Z. Zhang, K. Wang, G. Sheng, and G. Zhang, "Angle estimation and mutual coupling self-calibration for ULAbased bistatic MIMO radar," Signal Processing, vol. 144, no. 3, pp. 61-67, 2018.

[6] S. Hong, Y. Zhang, Y. Wang, H. Gu, G. Gui, and H. Sari, "Deep learning-based signal modulation identification in OFDM systems," IEEE Access, vol. 7, pp. 114631-114638, 2019.

[7] M. Liu, J. Chen, B. Li, and J. Li, "Fractional frequency offset estimation for OFDM systems in non-cooperative communication," China Communications, vol. 13, no. 9, pp. 65-71, 2016.

[8] G. Gui, H. Huang, Y. Song, and H. Sari, "Deep learning for an effective nonorthogonal multiple access scheme," IEEE Transactions on Vehicular Technology, vol. 67, no. 9, pp. 8440-8450, 2018.

[9] W. Li, H. Liu, Y. Wang, Z. Li, Y. Jia, and G. Gui, "Deep learning-based classification methods for remote sensing images in urban built-up areas," IEEE Access, vol. 7, pp. 36274-36284, 2019.

[10] H. Huang, W. Xia, J. Xiong, J. Yang, G. Zheng, and X. Zhu, "Unsupervised learning based fast beamforming design for downlink MIMO," IEEE Access, vol. 7, no. 1, pp. 7599-7605, 2018.

[11] F. Meng, P. Chen, L. Wu, and X. Wang, "Automatic modulation classification: a deep learning enabled approach," IEEE Transactions on Vehicular Technology, vol. 67, no. 11, pp. 10760-10772, 2018.

[12] W. Xie, S. Hu, C. Yu, P. Zhu, X. Peng, and J. Ouyang, "Deep learning in digital modulation recognition using high order cumulants," IEEE Access, vol. 7, pp. 63760-63766, 2019.

[13] M. W. Aslam, Z. Zhu, and A. K. Nandi, "Automatic modulation classification using combination of genetic programming and KNN," IEEE Transactions on Wireless Communications, vol. 11, no. 8, pp. 2742-2750, 2012.

[14] H. Wang, L. Xu, Z. Yan, and T. A. Gulliver, "Low-complexity MIMO-FBMC sparse channel parameter estimation for industrial big data communications," IEEE Transactions on Industrial Informatics, vol. 17, no. 5, pp. 3422-3430, 2021.

[15] D. Kong, X.-G. Xia, P. Liu, and Q. Zhu, "MMSE channel estimation for two-port demodulation reference signals in new radio," Science China Information Sciences, vol. 64, pp. 169303:1-169303:2, 2021.

[16] C. Lélé, P. Siohan, and R. Legouable, "2 dB better than CPOFDM with OFDM/OQAM for preamble-based channel estimation," in Proceedings of the IEEE International Conference on Communication, pp. 1302-1306, Xiamen, China, May 2008.

[17] C. Lélé, J.-P. Javaudin, R. Legouable, A. Skrzypczak, and P. Siohan, "Channel estimation methods for preamble-based OFDM/OQAM modulations," in Proceedings of the European Wireless Conference, pp. 59-64, Delft, Netherlands, March 2007.

[18] D. Kong, X. Zheng, Y. Zhang, and T. Jiang, "Frame repetition: a solution to imaginary interference cancellation in FBMC/ OQAM systems," IEEE Transactions on Signal Processing, vol. 68, pp. 1259-1273, 2020.

[19] D. Kong, J. Li, K. Luo, and T. Jiang, "Reducing pilot overhead: channel estimation with symbol repetition in MIMO-FBMC systems," IEEE Transactions on Communications, vol. 68, no. 12, pp. 7634-7646, 2020. 